\title{
Opinion
}

\section{Cancer Dynamics}

\author{
Daniel Gandia, MD ${ }^{1 *}$; Cecilia Suárez, PhD $^{2}$ \\ 'Clinical Oncologist IQVIA, Hematology and Oncology IQVIA, Medical Strategy and Science, Therapeutic Science \& Strategy Unit, Ing. Butty $2759^{\text {th }}$, CI00I CABA, Argentina \\ ${ }^{2}$ Complex Systems Lab, INFIP, FCEyN, Universidad de Buenos Aires - CONICET, Buenos Aires, Argentina
}

\section{*Corresponding author}

Daniel Gandia, MD

Clinical Oncologist IQVIA, Hematology and Oncology IQVIA, Medical Strategy and Science, Therapeutic Science \& Strategy Unit, Ing. Butty $2759^{\text {th }}$, CI00I CABA, Argentina; Phone. 54II 4I32 65I6; E-mail: Daniel.Gandia@iqvia.com

\author{
Article information \\ Received: March 23 $3^{\text {rd }}$, 2020; Revised: April 23 $3^{\text {rd }}$, 2020; Accepted: April 30 \\ Cite this article \\ Gandia D, Suarez C. Cancer dynamics. Cancer Stud Mol Med Open J. 2020; 6(I): I-4. doi: 10.17| 40/CSMMOJ-6-I30
}

$\mathrm{E}$ ven though cancer researchers nowadays are trying to develop many key cellular pathways susceptible to be tackled by therapeutic approaches, we are far from being able to consider some cancers as curable diseases in general terms. From a pharmacodynamic point of view, the main potential molecular targets involved are driver oncogenes, tumor suppressor genes, growth factors and their receptors, transcription factors, tyrosine kinases, and cell adhesion molecules. If we consider possible targets at higher organization levels, we can intervene in aspects such as chromatin organization, tumor microenvironment, angiogenesis, apoptosis, senescence, mitochondria metabolism, and immune pathways.

On the other hand, the Pan-cancer genome and transcriptome have been analyzed in more than 1500 pediatric leukemias and solid tumors. This analysis evidenced, for example, that pediatric central nervous system tumors are genetically different from their adult counterparts. In the meantime, whole-genome sequencing analysis of more than 2,500 tumors has already been done which is a continuing evolving genomic effort. A reconstruction of the life history and evolution of mutational processes and driver mutation sequences of 38 types of tumors have been throughout analyzed in detail. This unveiled different chromosomal and gene abnormalities are present at different stages of the tumorigenesis process. ${ }^{1}$

All the aforementioned means an essential part of the fascinating cancer machinery and the new therapeutics that have been designed for many of the cellular targets are described with different levels of success. Regarding oncogenes, it's noteworthy that the RAS family, one of the most important and frequently mutated in many tumors, has not received a "knock-out" yet, turning this family as the "Muhammad Ali" of this cancer story.

Some recently developed novel drugs such as AMG 510, that is directed to the G12C niche within K-Ras, are being tested nowadays in the clinics with promising results in some tumors such as advanced non-small-cell lung cancers. ${ }^{3}$ Other cellular-related Ras pathways such as phosphatidylinositol-3-kinase (PI3K/ AKT) and mammalian target of rapamycin (mTOR) have their own designed drugs that proved their worth, though to a lesser extent, in some tumor clinical settings. ${ }^{4}$

Regarding tumor suppressor genes, things tend to become more difficult and challenging for the moment, as a putative therapeutic agent must restore something that is missing or grossly inefficient. P53, one of the deoxyribonucleic acid (DNA) guardians and most well-known tumor suppressor genes, is being part of intensive research in relation to drugs that can "do something" at this level. ${ }^{5}$ Nevertheless, more than one hundred different P53 mutations yet described tell us about its complexity.

Growth factor family and their related receptor pathways are other branches of intensive research with clinical success. The discovery of the human epidermal growth factor receptor (HER) family, a transmembrane epidermal-growth-factor (EGF) family of receptors, has been one of the outstanding basic cancer research achievements of the past three decades with immediate clinical translation impact: HER-2 has been found to be completely blocked by Herceptin (Trastuzumab), a monoclonal antibody, combined with chemotherapy, has saved many lives in HER-2 positive early and advanced breast cancer.

Other "cousins" of Herceptin are also already in the clinical setting, proving their efficacy and medical benefits: Pertuzumab, Trastuzumab emtansine, Lapatinib, etc. Another classic EGF receptor family blocker, Cetuximab, has rendered good clinical results when combined with chemotherapy in wild-type Kirsten rat sarcoma viral oncogene homolog (K-RAS) advanced 
and recurrent colorectal and squamous head and neck cancers. ${ }^{7,8}$

Tyrosine kinases (TKS) are other neuralgic druggable targets. Anticancer agents of novel molecular design types, the so-called "small molecules", can act as inhibitors of the uni-kinase or multi-kinase TKS points and their downstream pathways (mTOR, AKT). The "first in class" of this kind of inhibitors was Imatinib, "the drug of the new millennium" (launched in 2001), specifically designed to target the TK domain within the Philadelphia chromosome $B C R-A B L$ region in chronic myeloid leukemias. This drug is used to treat, with outstanding results (overall survival of more than 15-years), refractory patients previously considered as chemo-reluctant. ${ }^{\text {? }}$

A very recent review ${ }^{10}$ emphasizes that there are currently more than fifty small molecules that function as TKS inhibitors used to treat a broad spectrum of malignancies. Renal clear cell carcinoma is a type of tumor that has obtained an important clinical benefit from these kinds of compounds (Sunitinib, Pazopanib, Sorafenib). Considering other druggable targets, transcription factors are at present a current area of new and intensive research, with many transcription factor inhibitors in early clinical phases of development. ${ }^{11}$

Chromatin organization/reorganization in cancer is complex, and its implications in euchromatin/heterochromatin rate, "readable" genes, nucleosome composition and cell epigenetics, are other future challenging roads to go through. This led to the appearance of epigenetic therapy. Hypomethylating agents are chromatin-targeted drugs with known beneficial effects in hematologic premalignancy as myelodysplastic syndromes and some blood cancers such as acute myeloid leukemia (AML). ${ }^{12}$

But cancer also develops many more alterations at higher organization levels than the molecular one. At the cellular level, this means self-suffiency to growth signals, evasion from growth-inhibitory ones, evasion from apoptosis, limitless replicative potential, survival after cell-cell detachment, altered energy metabolism, and acquisition of other special capabilities. Among these last ones, cell motility, invasiveness, and survival during the travel and settlement in new and not-friendly metastatic niches. ${ }^{13}$ Tumor cells may also acquire not only immunologic escape abilities but also the power of inducing T-cell death. At the tissue level, there are marked alterations in fluid dynamics, as normal plasma passage to tissues is subjected to more interstitial pressure constraints and a new set of capillaries developed through neoangiogenesis.

For all these pharmacodynamic roads to target, only the angiogenesis inhibition and immune-directed therapies have proved to be important therapeutic tools today in the clinical arena. Angiogenesis is key in tumor feeding and an inflection point to tumor malignization. Nutrients and oxygen are delivered to the cancer cells from new modified "tube formation": an abnormal and fenestrated capillary network that results in heterogeneous and rather chaotic tumor irrigation that difficult the administration of therapeutic agents.
One of the first "mammoths", able to disrupt the development of these new capillaries by targeting the vascular endothelial growth factor receptor (VEGFR), was the monoclonal antibody Bevacizumab. With this drug, the treatment of advanced and non-advanced colorectal, cervical and ovarian cancers became a reality. At present, continuous research in this area has developed fewer toxic antiangiogenics ("less bleeding ones", mainly of the small-molecules type) that are already in the clinical arena (already mentioned "renal cancer drugs" are some examples). ${ }^{10,14}$

Cancer immunology today highlights its relevance as a "new cancer science". George Bernard Shaw was right, in "The doctor's dilemma" play from 1906, when one of the actors states: "there is at bottom only one genuine scientific treatment for all diseases and that is to stimulate phagocytes". This was unconsciously referring to the innate immunology. The same type of discovery was made by Metchnikoff, "the father of phagocytosis", nearly at the same time. ${ }^{15}$

There are different types of these kinds of immune therapies, but at present, the most explored and promising ones are the checkpoint inhibitors (CPIs). ${ }^{16}$ Monoclonal antibodies such as Ipilimumab, Nivolumab, and Pembrolizumab are compounds of this class, leading to a new therapeutic concept in cancer immunology. These compounds allow natural killer cells "to eat tumors", strictly speaking, inhibiting or eliminating the negative immune regulation induced by the tumor. ${ }^{17}$

CPIs demonstrated a significant increment in advanced melanoma survival, with important visceral responses and achieving, in some cases, complete responses, "the cure". This made to recall the prior days as a Resident in Oncology. At that time, in 1987, Dr. Steven Rosenberg's discovery was fascinating such as high-dose interleukin-2 treatment delivered in the Intensive Care Unit that rendered some patients with peculiar tumor responses, mainly in renal cancers and melanoma. ${ }^{18}$ And when complete, they were long-lasting! This was, by that time, the best immunotherapy ever known. Unfortunately, it was toxic. The cytokine and white blood cell storm induced by these high doses of interleukin-2 was in some cases the cause of the patient's death. Hence, this treatment was avoided.

Nowadays, CPIs such as Pembrolizumab are standard of care in different tumor settings as head and neck cancer, renal cell carcinoma, non-small cell lung cancer, liver cancer, and microsatellite instability- (MSI) agnostic tumors. More "complexdesigned" immunotherapies are both in early and advanced developmental phases, such as novel vaccines and oncolytic therapies with adenoviruses. Finally, chimeric antigen receptor (CAR) T-cell therapy is a newly designed concept that has changed the natural history of some advanced refractory blood cancers, yet to prove its worth with solid tumors. ${ }^{19}$

Regarding higher organization levels like tumor tissue structure and pathophysiology, other complex factors of tumorigenesis came up to focus, such as cell de-differentiation, epithelial-mesenchymal transitions, tumor mutation rate, clonal 
expansion rates, and complex and dynamic cellular interactions. Among these, different competitions and cooperations take place between tumor clones, tumor cells from different differentiation stages (for example, proliferating tumor cell/tumor stem cell rate) and between tumor cells and other cells from the microenvironment. $^{20,21}$

Indeed, the whole tumor microenvironment may be completely altered, as previously normal cells from the stroma (fibroblasts, adipocytes) may have been converted by the tumor in tumor-associated cells that begin to work for its maintenance. ${ }^{22}$ All these mentioned cellular processes lead to a different level of tumor complexity and heterogeneity, becoming this last one, the main cause of treatment resistance, and tumor recurrence. This converts all these phenomena into a fascinating and challenging druggable target.

From an integrative point of view, cancer involves dynamic genetic alterations as well as tumor-host interactions that determine the destiny of the tumor and the host. ${ }^{23}$ Among the last ones, and settled at the whole-organism organization level, tumorigenesis begins with a great demand for nutrients from the tumor that cannot be satisfied by the host. This causes tumor cells to reside in a dismal environment that induces glycolytic metabolic shifts; angiogenesis; and force cells to invade, metastasize, and undergo necrosis. Tumor necrosis, particularly in metastases, leads to non-specific systemic inflammation, which is the major cause of cancer-related mortality.

In the end, in front of a cancer patient and considering all the aforementioned scenarios with a critical eye, we can summarize them in four ones. First, which are the tumor oncogenic drivers and which the more irrelevant mutations. Second, which are the main underlying mechanism/s of tumor progression. Third, how is the inner cell dynamics of the tumor? And fourth, how is the tumor-host interaction. All these four scenarios can help us, not only for making better use of old and novel therapeutic agents in use but also with the more complex coming ones that will specifically target cellular dynamics.

\section{CONFLICTS OF INTEREST}

The authors declare that they have no conflicts of interest.

\section{REFERENCES}

1. Gerstung M, Jolly C, Leshchiner I, Dentro SC, Gonzalez S, Rosebrock D, et al. The evolutionary history of 2.658 cancers. Nature. 2020; 578: 122-128. doi: 10.1038/s41586-019-1907-7

2. Stephen AG, Esposito D, Bagni RK, McCormick F. Dragging Ras back in the ring. Cancer Cell. 2014; 25: 272-281. doi: 10.1016/j. ccr.2014.02.017

3. Herbst R, SchlessingerJ. 'Undruggable' cancer protein targeted. Nature. 2019; 575: 294-295.

4. Goncalves MD, Hopkins BD, Cantley LC. Phosphatidylinosi- tol-3-kinase growth disorders and cancer. N Engl J Med. 2018; 379(21): 2052-2062. doi: 10.1056/NEJMra1704560

5. Muller P, Vousden K. Mutant p53 in cancer: New functions and therapeutic opportunities. Cancer Cell. 2014; 25: 304-317. doi: 10.1016/j.ccr.2014.01.021

6. Hayes DF. HER2 and breast cancer: A phenomenal success story. N Engl J Med. 2019; 381(13): 1284-1286. doi: 10.1056/ NEJMcibr1909386

7. Dekker E, Tanis PJ, Vleugels JLA, Kasi PM, Wallace MB. Colorectal cancer. The Lancet. 2019; 394: 1467-1480. doi: 10.1016/ S0140-6736(19)32319-0

8. Chow LQM. Head and neck cancer. N Engl J Med. 2020; 382(1): 60-72. doi: 10.1056/NEJMra1715715

9. Claudiani S, Apperley J. The argument for using imatinib in CML. Hematology Am Soc Hematol Educ Program. 2018; 2018(1): 161-167. doi: 10.1182/asheducation-2018.1.161

10. Bedard P, Hyman D, Davids M, Siu L. Small molecules, big impact: 20 years of targeted therapy in oncology. Lancet. 2020; 395: 1078-1088. doi: 10.1016/S0140-6736(20)30164-1

11. Stathis A, Bertoni F. BET proteins as targets for anticancer treatment. Cancer Discov. 2018; 8(1): 24-36. doi: 10.1158/21598290.CD-17-0605

12. Mei M, Aldoss I, Marcucci G, Pullarkat V. Hypomethylating agents in combination with Venetoclax for acute myeloid leukemia: Update on clinical trial data and practical considerations for use. Am J Hematol. 2019; 94(3): 358-362. doi: 10.1002/ajh.25369

13. Hanahan D, Weinberg R. Hallmarks of cancer: The next generation. Cell. 2011; 144: 646-674. doi: 10.1016/j.cell.2011.02.013

14. Qiao Y, Dsouza C, Matthews AA, Jin Y, He W, Bao J, et al. Discovery of small molecules targeting GRP78 for antiangiogenic and anticancer therapy. Eur J Med Chem. 2020; 193: 112228. doi: 10.1016/j.ejmech.2020.112228

15. Teti G, Biondo C, Beninati C. The phagocyte, Metchnikoff, and the foundation of immunology. Microbiol Spectr. 2016; 4(2). doi: 10.1128/microbiolspec.MCHD-0009-2015

16. Ribas A, Wolchok J. Cancer immunotherapy using checkpoint blockade. Science. 2018; 359(6382): 1350-1355. doi: 10.1126/science.aar4060

17. Allison JP, Honjo T. Press release: The Nobel Prize in Physiology or Medicine 2018. nobelprize Web site. https://www.nobelprize.org/prizes/medicine/2018/press-release/. Accessed March 22, 2020.

18. Rosemberg S, Lotze M, Muul L, Chang A, Avis F, et al. A progress report on the treatment of 157 patients with advanced 
cancer using lymphokine-activated killer cells and interleukin-2 or high-dose interleukin-2 alone. N Engl J Med. 1987; 316(15): 88997. doi: 10.1056/NEJM198704093161501

19. Mohanty R, Chowdhury CR, Arega S, Sen P, Ganguly P, Ganguly N. CAR T-cell therapy: A new era for cancer treatment. Oncol Rep. 2019; 42(6): 2183-2195. doi: 10.3892/or.2019.7335

20. Korolev K, Xavier J, Gore J. Turning ecology and evolution against cancer. Nat Rev Cancer. 2014; 14: 371-380. doi: 10.1038/ nrc3712
21. Powell K. Survival of the fittest cells. Nature. 2019; 574: 310312. doi: 10.1096/fj.08-108985

22. Muppalla J, Muddana K, Dorankula S, Thokala M, Pasupula A. Microenvironment: A role in tumour progression and prognosis. J Clin Diagn Res. 2013; 7(9): 2096-2099. doi: 10.7860/ JCDR/2013/6619.3419

23. Luo G, Liu N. An integrative theory for cancer. Int J Mol Med. 2019; 43: 647-656. doi: 10.3892/ijmm.2018.4004 